\title{
Metabolic Reprogramming Promotes Metastasis of Acidosis-Adapted Pancreatic Ductal Adenocarcinoma Cells Via AMPK/YAP/MMP1 Axis
}

\section{Siyuan Chen}

The Second Affiliated Hospital of Chongqing Medical University

\section{Bo Ning}

The Second Affiliated Hospital of Chongqing Medical University

Jinwen Song

Chinese PLA General Hospital Fifth Medical Center South Campus

\section{Zihan Yang}

City University of Hong Kong

\section{Li Zhou}

The Second Affiliated Hospital of Chongqing Medical University

\section{Zhiji Chen}

The Second Affiliated Hospital of Chongqing Medical University

\section{Linhong Mao}

The Second Affiliated Hospital of Chongqing Medical University

\section{Hongtao Liu}

The Second Affiliated Hospital of Chongqing Medical University

\section{Qingliang Wang}

The Second Affiliated Hospital of Chongqing Medical University

\section{Song He}

The Second Affiliated Hospital of Chongqing Medical University

Zhihang Zhou ( $\square$ zhouzhihang@cqmu.edu.cn )

The Second Affiliated Hospital of Chongqing Medical University https://orcid.org/0000-0003-13569872

\section{Primary research}

Keywords: Acidic microenvironment, PDAC, metastasis, MMP1, Hippo signaling, AMPK

Posted Date: November 23rd, 2021

DOI: https://doi.org/10.21203/rs.3.rs-1068360/v1 
License: (c) (i) This work is licensed under a Creative Commons Attribution 4.0 International License. Read Full License 


\section{Abstract \\ Background}

Pancreatic ductal adenocarcinoma (PDAC) is one of the most lethal cancers. Acidic microenvironment, namely acidosis, is closely related to cancer development and progression, but the regulatory mechanism remains to be elucidated.

\section{Methods}

We generated acidosis-adapted PDAC cells (PANC-1 and SW1990) by continuously culturing under pH 6.5 medium. Metabonomic assay (LC-MS and LC-MS) was used to detect the metabolites. Transcriptomic microarray was applied to reveal the gene expression profile. The in vivo metastatic potential was assessed by tail vein injection. Wound healing assay and transwell assay were applied to evaluate cell migration and invasion capacity. CCK8 and colony formation were performed to determine cell proliferation. Flow cytometry was used to test cell cycle and apoptosis.

\section{Results}

These acidosis-adapted PDAC cells had stronger invasion and migration ability, accompanied by increased EMT markers expression. In addition, the proliferation rate of acidosis-adapted PDAC cells increased while apoptosis decreased under acidic medium. Metabonomic analysis showed that the glycolysis and AMP was decreased, and ATP was increased in acidosis-adapted cells. The metabolic shift led to the inactivation of AMPK. Transcriptomic analysis revealed that the differentially expressed genes in acid-adapted cells were enriched in extracellular matrix modification, Hippo signaling and others. MMP1, a matrix metalloproteinase, was the most upregulated gene in acidosis-adapted cells, mediated by the YAP/TAZ pathway. Knockdown of MMP1 significantly reduced invasion and metastasis capacity of acidosis-adapted cells. The AMPK activator MK8722 could reduce both YAP and MMP1 level in acidosis-adapted PDAC cells.

\section{Conclusion}

Altogether, the present study showed that the metabolic reprogramming towards elevated ATP production promotes invasion and metastasis of acidosis-adapted PDAC cells via depressing AMPK/Hippo signaling, thus upregulating MMP1.

\section{Background}

To adapt to the adverse living environment caused by the unlimited proliferation, the metabolic mode of tumor cells presents a background dependent flexibility ${ }^{1}$. Tumor cells tend to aerobic glycolysis to meet 
rapid proliferation, which generates a large amount of lactic acid. In addition, proton and carbon dioxide are respectively produced in the process of ATP consumption and oxidative phosphorylation. All these acidic materials are expelled into the extracellular microenvironment and retained in this space due to the hypoperfusion, leading to $\mathrm{pH}$ value of tumor microenvironment decreased to $6.4-7$, namely acidosis ${ }^{2}$. Acidosis is a selective pressure for tumor cells, and the one that survive have additional survival advantages, such as enhanced proliferation, metastasis and drug resistance ${ }^{3}$. Hepatocellular carcinoma cells proliferation and metastasis increased under acidosis condition explained well for this phenomenon ${ }^{4}$. Furthermore, Faes $\mathrm{S}$ et al. revealed that the acidic tumor microenvironment made mTORC1 inhibitors ineffective ${ }^{5}$. Moreover, lower $\mathrm{PH}$ value promotes malignant glioma ${ }^{6}$, breast cancer ${ }^{7}$, colon cancer ${ }^{8}$, lung cancer ${ }^{9}$ progression. Our previous work found that the acid sensing ion channel ASIC2 promotes colorectal cancer cell invasion under acidosis ${ }^{10}$, and acid-adapted colon cancer cells have stronger metastasis capacity ${ }^{11}$. As the widely recognized pro-tumor effect, acidosis has been applied as a marker for prognosis prediction ${ }^{12}$ and treatment target ${ }^{13}$. However, the underlying mechanism of acidosis should be further demonstrated, especially for chronic acidosis.

The acidic microenvironment can also in turn induce a metabolism shift. Minnan Zhao and colleagues found that acidosis-adapted colorectal cancer cells (HCT15, HCT116 and LoVo) maintain a higher level of reduced glutathione to reduce the acid-induced reactive oxygen species via the upregulation of CD44 and glutathione reductase (GSR) ${ }^{14}$. Additionally, the acidosis-adapted colorectal cancer cell (HCT116), cervical cancer cell ( $\mathrm{SiHa}$ ) and pharynx squamous cell carcinoma cell (FaDu) all exhibited a turnover from glycolysis to glutamine metabolism, which was mediated by HIF $2 \mathrm{a}^{15}$. The same research group further revealed that acidic microenvironment profoundly reprograms the metabolism of these cancer cells toward fatty acid oxidation by inducing histone deacetylation and downregulating acetyl-CoA carboxylase ACC2 ${ }^{16,17}$. As metabonomics technology developed, it can reveal significant metabolic changes under acute or chronic acidosis. Recently, it was reported that acute acidic treatment $(\mathrm{pH} 6.8$ for 36 hours) increased the de novo purine nucleotide biosynthesis activity in glioma stem cells by upregulating glucose-6-phosphate dehydrogenase G6PD or H6PD expression ${ }^{18}$. AMP-activated protein kinase (AMPK) is an energy sensor that monitors the AMP:ADP:ATP ratio in eukaryotic cells. It has generally been considered a tumor suppressor as it is a putative substrate of tumor suppressor, LKB1 ${ }^{19}$. AMPK phosphorylates angiomotin like 1 (AMOTL1), an adaptor protein in the Hippo-Yap pathway, and thus blocks Yes1 associated transcriptional regulator (YAP) activity, dampening cancer cells proliferation and survival ${ }^{20}$. Further work should be done to reveal the metabolic reprogramming under chronic acidosis and its correlation with tumor progression, especially in the context of pancreatic ductal adenocarcinoma (PDAC), one of the most lethal malignancies ${ }^{21}$.

In this study, we generated the acidosis-adapted PDAC cells by continuously culturing under $\mathrm{pH} 6.5$ medium treatment for 3 months. We found that cells proliferation and metastasis ability increased, and MMP1 expression was profoundly upregulated. Furthermore, glycolysis was significantly inhibited, and intracellular ATP level and other metabolites increased in acidosis-adapted PDAC cells. Inhibition of 
AMPK signaling by increased ATP level then increased the YAP and MMP1 expression, promoting metastasis. Our study demonstrated that metabolic reprogramming promotes metastasis of acidosisadapted PDAC cells via AMPK/YAP/MMP1 axis, enriching the mechanism by which acidic environment promotes tumor progression.

\section{Methods}

\section{Cell lines and cell culture}

Human pancreatic cancer cell lines PANC-1 and SW1990 were obtained from the American Type Culture Collection (Manassas, VA, USA), all the cells were identified by STR and without mycoplasma contamination. Cells were cultured in Dulbecco's Modified Eagle Medium PH 7.4 or PH6.5 (HyClone, Logan, UT, USA). All culture media were supplemented with 10\% FBS (Gibco, Rockville, MD, USA), 100 units $/ \mathrm{mL}$ penicillin, and $100 \mu \mathrm{g} / \mathrm{mL}$ streptomycin (HyClone). All cells were grown in a $37^{\circ} \mathrm{C}$ humidified atmosphere containing $5 \% \mathrm{CO}_{2}$.

\section{Metabonomic analysis}

The metabonomic analysis was done by Oebiotech (Shanghai, China). Briefly, $5 \times 10^{7}$ cells were broken up using ultrasonic homogenizer in mixture of methanol and water (1/4, vol/vol). L-2-chlorophenylalanine $(0.3 \mathrm{mg} / \mathrm{mL})$ dissolved in methanol was used as internal standard. The dried supernatant was then dissolved in pyridine containing $15 \mathrm{mg} / \mathrm{mL}$ methoxyamine hydrochloride. The samples were then subjected to LC-MS and GC-MS analysis. The obtained GC/MS raw data in .D format were transferred to .abf format via software Analysis Base File Converter for quick data retrieval. Then, data were imported into software MS-DIAL, which performs peak detection, peak identification, MS2Dec deconvolution, characterization, peak alignment, wave filtering, and missing value interpolation. Metabolite characterization is based on LUG database. The original LC-MS data were processed by software Progenesis QI V2.3 (Nonlinear, Dynamics, Newcastle, UK) for baseline filtering, peak identification, integral, retention time correction, peak alignment, and normalization. Main parameters of $5 \mathrm{ppm}$ precursor tolerance, $10 \mathrm{ppm}$ product tolerance, and $5 \%$ product ion threshold were applied. Compound identification were based on precise mass-to-charge ratio (M/z), secondary fragments, and isotopic distribution using The Human Metabolome Database (HMDB), Lipidmaps (V2.3), Metlin, EMDB, PMDB, and self-built databases to do qualitative analysis. Variable Importance of Projection (VIP) values obtained from the OPLS-DA model were used to rank the overall contribution of each variable to group discrimination. A two-tailed Student's T-test was further used to verify whether the metabolites of difference between groups were significant. Differential metabolites were selected with VIP values greater than 1.0 and $p$-values less than 0.05 .

\section{Clinical PDAC samples}

PDAC specimens were collected from 61 patients with PDAC from 2011 to 2015 at the Second Affiliated Hospital of Chongqing Medical University after informed consent was obtained from all patients. The 
patients did not receive chemotherapy or radiotherapy before surgery. The diagnoses of PDAC were made independently by at least two histopathologists. This study was carried out according to the principles of the Helsinki Declaration and approved by the Ethical Committee of the Second Affiliated Hospital of Chongqing Medical University ((2019)133).

\section{Lentivirus infection}

Lentiviruses carrying small hairpin RNA (shRNA) sequence of human MMP1 were purchased from Obio Company (Shanghai, China). Sequences for MMP1 shRNA and control were as follows: shRNA-1 (5'GCCTTCCAACTCTGGAGTAAT-3'), shRNA-3(5'- GCGTGTGACAGTAAGCTAACC-3') and control (5'AAACGTGACACGTTCGGAGAA-3'). Cells were planted in 24-well plates at $1 \times 10^{5}$ cells/well, lentivirus was added into the medium separately $(\mathrm{MOI}=20)$. The mediums were refreshed after $12 \mathrm{~h}$. Puromycin was used to screen the stable cells after $72 \mathrm{~h}$ of infection.

\section{RNA extraction and real-time quantitative PCR (qPCR)}

Total RNA was extracted from each group with TRIzol Reagent (Takara, Dalian, China). The cDNA was obtained by using PrimeScript RT Master Mix (Takara, Japan). 50ng of each cDNA was amplified as a template, and qPCR was performed in a CFX96 real-time PCR system (Bio-Rad, Hercules, CA, USA) with SYBR ${ }^{\circledR}$ Premix Ex TaqTM II kit (Takara, Japan). The PCR primers used are listed in supplementary table 1.

\section{mRNA expression microarray}

RNA from acidosis-adapted PANC-1 cells and parental cells was extracted as mentioned above. Then the RNA was incubated with Agilent mRNA+LncRNA microarray (Capitalbiotech, Beijing, China). The differentially expressed genes were screened by the criterion: fold change larger than 2 and $p$ value less than 0.05 . Then these genes were applied to enrichment analysis by KEGG and GO database.

\section{Tail vein injection model}

Briefly, $1 \times 10^{7}$ tumor cells (SW1990-NA-NC, SW1990-NA-shMMP1, SW1990-AA-NC, SW1990-AA-shMMP1)

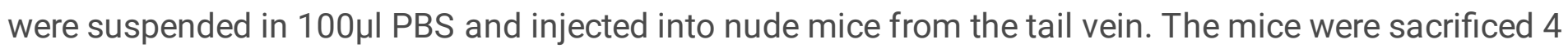
weeks after injection and the liver and lung were collected for pathological test. The lungs and livers were dissected into $5 \mathrm{~mm}$ thick pieces and each piece was subjected for dehydration, embedding. Pathological section with $4 \mu \mathrm{m}$ thickness were made and stained with H\&E. The metastatic foci were identified by a pathologist.

\section{Wound healing assay}

After cell fusion rate in the 6 -well plate reached more than $90 \%$, then remove the cell medium. A $10 \mu \mathrm{L}$ pipette tip was used to draw a straight line in the cells along the sterilized ruler. Cells were washed with PBS for 3 times. Cell migration was observed and recorded at $0 \mathrm{~h}$ and $24 \mathrm{~h}$. 


\section{Cell migration and invasion assays}

Twenty-four-well transwell chambers were used for this assay (BD Falcon, Franklin Lakes, NJ). Briefly, $3 \times 10^{4}$ cells were plated into each upper chamber with 8 - $\mu \mathrm{m}$ pores and cultured in $200 \mu \mathrm{L}$ serum-free DMEM. The lower chambers were filled with 500 $\mu \mathrm{L}$ complete DMEM medium. After incubation for 24 hours at $37^{\circ} \mathrm{C}$, cells that had migrated in the lower chambers were fixed with $4 \%$ polyformaldehyde and stained with $0.1 \%$ crystal violet. The number of cells was counted in 5 distinct areas at $\times 100$ magnification. The results represent the average cell number in 3 wells per cell line. Then, the upper surface of the polycarbonate filter was coated with 10\% Matrigel TM (BD Biosciences, Franklin Lakes, $\mathrm{NJ}$ ), and $5 \times 10^{4}$ cells were added to detect cell invasion. The other conditions were the same as those in the migration assay.

\section{Immunofluorescence assay}

Cells were fixed with $4 \%$ polyformaldehyde for $20 \mathrm{~min}$, and blocked with goat serum for $1 \mathrm{~h}$ at room temperature. Cells were then incubated with an anti-E-cadherin or $\beta$-actin antibody for $1 \mathrm{~h}$ at room temperature. After three washes in PBS, cells were incubated with a Cy3-conjugated secondary antibody (Beyotime, China) for $1 \mathrm{~h}$. The nuclei were stained with DAPI for $5 \mathrm{~min}$. Images were captured on a confocal microscope (Leica SP5, Germany).

\section{Cell proliferation assay}

Cells were plated in 96-well plates (2000 cells/well) in triplicate and measured using Cell Counting Kit-8 (CCK-8) (Dojindo Laboratories, Kumamoto, Japan). Cell proliferation was determined every $24 \mathrm{~h}$ for three days following the manufacturer's protocol. The absorbance at $450 \mathrm{~nm}$ was measured with a microplate reader (Bio-Rad, Hercules, CA, USA).

\section{Colony formation assay}

Long-term survival of cells was assessed by their ability to form colonies. 100 cells were seeded in a 96well plate. After 15 days, colonies were fixed and stained with $0.1 \%$ crystal violate (Invitrogen, Carlsbad, CA, USA) before counting.

\section{Cell cycle analysis}

For cell cycle detection, $1.0 \times 10^{6}$ cells were harvested and fixed with $75 \%$ ethanol at $4^{\circ} \mathrm{C}$ overnight after being washed with PBS. Then, cells were washed with PBS and stained with propidium iodide. FACSCalibur instrument (BD Biosciences, Franklin Lakes, NJ, USA) and CellQuest software were used to analyze cell cycle.

\section{Cell apoptosis assay}


Cells were obtained at a density of $1.0 \times 10^{6} \mathrm{cells} / \mathrm{mL}$ and washed by PBS, then incubated with reagents from the Annexin-V-FITC Apoptosis Detection Kit (Neobioscience, Shenzhen, China) according to the manufacturer's protocol. Then the cells were analyzed by FACS Vantage SE flow cytometer (BD Biosciences, San Jose, CA, USA).

\section{Immunohistochemistry (IHC)}

Following deparaffinization and rehydration, tissue samples ( $5 \mathrm{~mm}$ slices) were incubated in $0.3 \% \mathrm{H}_{2} \mathrm{O}_{2}$ in methanol for $30 \mathrm{~min}$ at $37^{\circ} \mathrm{C}$ to block endogenous peroxidase. The sections were then boiled in 10 $\mathrm{mmol} / \mathrm{L}$ citrate buffer $(\mathrm{pH} \mathrm{6.0)}$ for $2 \mathrm{~min}$ in an autoclave. tissue samples were incubated with anti-MMP1 (Abcam, UK) overnight at $4{ }^{\circ} \mathrm{C}$. MMP1 was detected using HRP-conjugated anti-rabbit secondary antibody (ZSGB-BIO, China) and visualized with DAB. The negative control only incubated with secondary antibody. The intensity of staining (brown color) was semi-quantitatively scored as follows: 1 , weak; 2 , medium; 3 , strong; and 4 , very strong. The percentage of maximally stained tumor cells in each section was recorded $(0,<5 \% ; 1,5-30 \% ; 2,30-50 \% ; 3,>50 \%)$. High expression of MMP1 was defined as a combined score for the intensity and area of staining that was larger than 6 . The results were verified by two pathologists independently.

\section{Western blotting}

Whole-cell lysates were extracted in a lysis buffer (Beyotime, Shanghai, China) and were separated by $10 \%$ SDS-PAGE, then transferred to a polyvinylidene fluoride membranes (Millipore, Billerica, MA, USA) by using a wet transfer apparatus (Bio-Rad, German). Blots were blocked in $5 \%(\mathrm{w} / \mathrm{v})$ skim milk for $2 \mathrm{~h}$ at room temperature and then incubated with antibodies against snail, Vimentin, MMP1 (Abcam, Cambridge, UK), P-TAZ, P-YAP, TAZ, YAP, AMPK, p-AMPK (Cell Signaling Technology, USA) and GAPDH (ZSGB-BIO, China) overnight at $4{ }^{\circ} \mathrm{C}$. The HRP-coupled anti-rabbit secondary antibody (ZSGB-BIO, China) was used at a final dilution of 1:1000, visualized with an enhanced chemiluminescence (ECL) detection system (Thermo Scientific, Waltham, MA, USA).

\section{Statistical analysis}

All values were presented as means \pm standard deviation. All data were obtained from at least three repetitions of each experiment. Prism 8.0 (GraphPad, USA) software was used to analyze the data. All data are shown as mean \pm SD. Student's t-test was used to analyze differences between two groups. Oneway ANOVA was used to compare three or more groups. Probability values less than 0.05 was considered statistically significant.

\section{Results}

\section{Reduction in AMP level activates AMPK signaling in acidosis-adapted PDAC cells}


Acidosis-adapted PDAC cells (PANC-1-AA and SW1990-AA) were obtained by culturing in DMEM acid medium of pH6.5 for three months, and the control cells were cultured in DMEM at pH7.4 (PANC-1-NA and SW1990-NA). Non-targeting metabonomic analysis, including LC-MS and GC-MS, was applied to reveal the metabolic changes in acidosis-adapted PDAC cells (SW1990 and PANC1). The GC-MS revealed that 99 and 69 metabolites were respectively changed in SW1990 (Fig1. a) and PANC1 cells (Supplementary Fig1. a). Accordingly, LC-MS revealed that 105 and 99 metabolites were respectively changed in SW1990 (Fig1. b) and PANC1 cells (Supplementary Fig1. b). The common decreased metabolites include adenosine monophosphate (AMP), fructose-6-phosphate (F6P), fructose-1-phosphate (F1P), fructose-1,6bisphosphate (FBP), glucose-6-phosphate (G6P) (Fig1. c). And the few common increased metabolites include glucose, metanephrine and myo-inositol (Fig1. d). The metabolites only increased in SW1990 cells include arginine, serine, glutamine, isoleucine, guanosine, ornithine, citrulline, glutathione, and valine (Fig1. d). The metabolites that were only increased in PANC1 cells includes phosphatidylcholine (PC), phosphosphingolipids (PS), glutaric acid, fumaric acid, tromethamine, testosterone, and methylamine (Fig1. d). The increased metabolites in PANC1 cells were enriched in choline metabolism in cancer, glycerophospholipid metabolism, glycine, serine and threonine metabolism and arginine/proline metabolism (Fig1. e). In contrast, the metabolites that were increased in SW1990 cells were mainly enriched in amino acid metabolism pathways such as arginine biosynthesis, glutathione metabolism, cysteine and methionine metabolism, valine/leucine/isoleucine biosynthesis (Fig1. f). As AMP was decreased in acidosis-adapted PDAC cells and the altered metabolites were related to energy production, we detect the intracellular ATP level and found that the ATP level was higher in acidosis-adapted cells (Fig1. g\&h). These results revealed that the PDAC cells have different compensatory metabolic ways to increase intracellular ATP level after inhibiting glycolysis. As the ATP/AMP ratio tightly regulates AMPK signaling, we detected the phosphorylation level of AMPK and found that the phosphorylation level of AMPK was lower in acidosis-adapted cells than parental cells (Fig1. i). Altogether, we found that the metabolic reprogramming in acidosis-adapted PDAC cells inhibited AMPK signaling.

\section{Acidosis-adapted PDAC cells exhibited enhanced migration and invasion ability.}

We then test the cellular behavior of acidosis-adapted cells. Wound healing test was used to detect the difference of migration ability between normal cultured PDAC cells and acidosis-adapted PDAC cells. We found that acid-adapted PDAC cells migrated faster than that of normal cells. The healing rate of PANC-1AA was about three times that of PANC-1-NA, while SW1990-AA was almost two times that of SW1990NA (Fig2. a\&b). Transwell assays showed that, compared with PANC-1-NA cells, the number of invasive cells of PANC-1-AA was obviously increased by about $50 \%$ (Fig2. c). Similar to PANC-1 cells, the invasive cells of SW1990-AA increased 30\% to SW1990-NA (Fig2. d). As is well known, epithelial-mesenchymal transition (EMT) is an essential factor in promoting invasion and metastasis, so we detected the expression of EMT markers. As exhibited in Fig2. e, mRNA level of N-cadherin, Vimentin, a-SMA, Snail, Zeb1 and Zeb2 were significantly increased in PANC-1-AA and SW1990-AA cells. Besides, Snail, Vimentin and $\beta$-catenin protein level increased as well (Fig2. $f \& g)$, but E-cadherin protein level decreased in acidadapted PDAC cells (Fig2. g). Therefore, acidosis-adapted PDAC cells exhibited enhanced migration and invasion ability. 
Previous studies revealed that acid-adapted colon cancer and cervical cancer cells could grow at a similar or higher rate in comparison to their parental cells ${ }^{22}$. Still, it is not clear whether chronic acidic microenvironment can affect the proliferation of PDAC cells. The results of CCK8 test showed that, compared with the NA cells in normal medium, the proliferation of PANC-1-AA and SW1990-AA cells were significantly improved in acidic medium (Fig3. a\&b). The colony formation assay also reflected similar results (Fig3. c\&d). Moreover, we compared the cell cycle of normal cells and acidosis-adapted cells. The number of PANC-1-AA cells in S phase was significantly more than that in PANC-1-NA cells, but the number of $\mathrm{G} 1$ phase cells was markedly reduced. There was no significant difference in the number of $\mathrm{G} 2$ phase cells between NA and AA cells. In SW1990 cells, the number of acidosis-adapted cells in S phase was more than that in normal culture, but there was no significant difference in other phases (Fig3. e\&f). All cell cycle pictures of above cells were in Supplementary Fig2. On the contrary, the apoptosis of PANC1-AA and SW1990-AA cells was less than that of PANC-1-NA and SW1990-NA cells (Fig3. g\&h). Therefore, acidosis-adapted cells have enhanced proliferation and less apoptosis than parental cells.

\section{MMP1 was significantly upregulated in acidosis-adapted PDAC cells}

We analyzed the differentially expressed genes of PANC-1-NA and PANC-1-AA cells by microarray technology. We found that MMP1, ASIC2 and NANOG expression were significantly upregulated in PANC1-AA cells, while the expression of LONRF2, BMP4 and COL5A1 were markedly decreased. Among them, the most obviously changed gene was MMP1 (Fig4. a). Interestingly, the glucose transporter SLC2A12 was also significantly upregulated in PANC-1-AA cells (Supplementary table 2), implying the redirection of increased glucose from glycolysis to pentose phosphate pathway, which provides the substrates for nucleic acid, fatty acids, sterols, nucleotides and non-essential amino acids synthesis ${ }^{23}$. GO analysis showed that the main difference between PANC-1-NA and PANC-1-AA cells were in cell adhesion and extracellular matrix organization (Fig4. b). qPCR and WB assays showed that both mRNA and protein level of MMP1 were increased either in PANC-1-AA cells or in SW1990-AA cells (Fig4. C).

MMP1 can degrade a variety of collagen and other extracellular matrix ${ }^{24}$. MMP1 expression level is increased in various malignant tumors and is negatively correlated with prognosis ${ }^{25}$. TCGA database showed that the expression of MMP1 in most cancer tissues was significantly higher than that in adjacent tissues (Fig4. d). However, the relationship between MMP1 and pancreatic cancer is rarely studied, especially in the acidic microenvironment of pancreatic cancer. We detected the expression of MMP1 in tissues of 61 patients with PDAC by immunohistochemistry (Fig4. e), and found the higher the expression of MMP1, the worse the prognosis (Fig4. f). The tumor size was larger in the MMP1 ${ }^{\text {high }}$ group than MMP1 ${ }^{\text {low }}$ (Fig4. g). Consistently, bioinformatic analysis revealed that the expression level of MMP1 was correlated with advanced tumor stage (Fig4. h) and poor prognosis (Fig4. i). Pathological characteristics of clinical samples revealed that high expression of MMP1 is associated with metastasis and acidic marker CA9 and acid sensing ion channels ASIC1 and ASIC2 (Table 1). These results showed 
that elevated MMP1 expression might at least partially mediate the enhanced invasion capacity of acidosis-adapted PDAC cells.

\section{Knock-down of MMP1 impairs invasion and metastasis of acidosis-adapted PDAC cells}

Database analysis (https://hgserver1.amc.nl) showed that the expression of MMP1 was positively correlated with acidic markers such as CA9 (Carbonic Anhydrase 9) $(P<0.0001)$, MCT1 (monocarboxylate transporter 1) $(P<0.0001)$ and MCT4 (monocarboxylate transporter 4) $(P=0.0004)$, and Lamp2 (Recombinant Lysosomal Associated Membrane Protein 2$)$ to a lesser degree $(P=0.059)$ in PDAC tissues (Supplementary Fig3). These results suggest that MMP1 is closely related to the acidic microenvironment. We knocked down the expression of MMP1 by interfering with lentivirus in PANC-1 and SW1990 cells (Fig5. a\&b), and found the invasion ability of acidosis-adapted cells was significantly reduced even in acidic environment, and the number of invasive cells decreased significantly compared with normal cultured cells (Fig5. c\&d). The in vivo impact of MMP1 was evaluated with tail vein injection model. The results showed the enhanced metastatic potential of acidosis-adapted PDAC cells compared to parental cells, and knocking down MMP1 expression significantly depressed AA cells in vivo metastasis (Fig5. e\&f). Thus, MMP1 plays a critical role in mediating the enhanced invasion and metastasis of acid-adapted PDAC cells.

\section{Activation of YAP/TAZ signaling upregulated MMP1 in acidosis-adapted PDAC cells}

In order to explore the regulatory mechanism of the acidic microenvironment of MMP1 in PDAC cells, we reviewed the microarray results and found that Hippo signaling pathway was significantly changed (Fig6. a). We detected the expression and activation of YAP and TAZ, the key molecules of Hippo pathway. The results showed that expression levels of YAP and TAZ in PANC-1-AA and SW1990-AA cells were significantly higher than those in PANC-1-NA and SW1990-NA cells (Fig6. b). When YAP/TAZ is phosphorylated, their activities are inhibited. Non-phosphorylated YAP/TAZ will enter the nucleus as cotranscription factors and combine with TEADs to promote oncogene transcription ${ }^{26}$. Thus, we chose YAP/TAZ inhibitor ML-7 to block their activities. After treatment with ML-7, the activation level of YAP and TAZ were significantly reduced, while the expression of MMP1 and Snail in PANC-1-AA and SW1990-AA cells were significantly decreased (Fig6. C). Transwell assays showed that the invasion capacity of PANC1-AA and SW1990-AA cells was reduced by about $50 \%$ after being treated with ML-7 (Fig6. d). The results of the scratch test were consistent with those of transwell (Fig6. e). Transwell and wound healing images were showed in Supplementary Fig. 4. In addition, YAP/TAZ inhibitor also significantly inhibited the proliferation of acid-adapted cells, compared with NA cells (Fig6. f). Moreover, we found a positive correlation between the expression of YAP and MMP1 in PDAC tissues (Supplementary Fig5). There is a predicted binding site of TEAD4 in the promoter region of MMP1, which is a recognized YAP/TAZ effector molecule ${ }^{27}$ (Fig6. g). We treated PDAC cells with different concentrations of AMPK inhibitor MK8722 and screened the optimal working concentration of $1 \mu \mathrm{M}$ (Supplementary Fig6). MK8722 treatment could dramatically down-regulate both YAP and MMP1 (Fig6. h). These results indicated that the inactivation of AMPK/Hippo signaling mediates the augmented MMP1 expression. 


\section{Discussion}

Studies have shown that tumor cells prefer forming acidic microenvironment because of high metabolic activity and insufficient perfusion. Tumor cells adapted to acidic microenvironment exhibited aggressive phenotype, which is achieved by acid-induced cell motility, extracellular matrix degradation, decreased immune responses and modified signaling pathways ${ }^{28}$. However, its specific regulatory mechanism has not been fully clarified. Our experiments elucidated that glycolysis was significantly inhibited, while intracellular ATP, glucose and other metabolites were increased, in acidosis-adapted PDAC cells. This metabolic reprogramming leads to AMPK inactivation. It should be noted that the glucose transporter SLC2A12 (GLUT12) rather than SLC2A1 (GLUT1) was also upregulated in acidosis-adapted PDAC cells. GLUT12 was required for maximal androgen-mediated glucose uptake and cell growth in prostate cancer cells (LNCaP and VCaP) ${ }^{29}$. In addition, GLUT12 is highly expressed in triple-negative breast cancer (TNBC) samples and promotes TNBC tumor growth and metastasis in vitro and in vivo through regulating aerobic glycolysis ${ }^{30}$. This evidence implies that acidosis-adapted uptake more glucose to fuel their aggressive activities.

Although the intracellular glucose was elevated in acidosis-adapted PDAC cells, it was not used for glycolysis due to the many intermediate products of glycolysis were reduced. Pentose phosphate pathway (PPP) is the other way for glucose catabolism. The PPP branches after the first step of glycolysis and consumes the intermediate glucose 6-phosphate (G6P) to generate fructose 6-phosphate (F6P) and glyceraldehyde 3-phosphate (G3P). The PPP supplies NADPH and ribose5-phosphate (R5P) instead of ATP ${ }^{23}$. These two metabolites are vital for cell survival and proliferation. R5P is a building block for nucleic acid synthesis. NADPH is the reducing power required to synthesize fatty acids, sterols, nucleotides and non-essential amino acids ${ }^{31}$. Moreover, NADPH mediates oxidized glutathione (GSSG) conversion to reduced glutathione (GSH) via glutathione reductase to reduce the reactive oxygen species. Recently, the PPP has been reported to enhance the chemoresistance of breast cancer cells ${ }^{32}$. We have reason to propose that the glucose was re-directed to PPP as the increase in intracellular glucose, amino acids, phosphatidylcholine, phosphosphingolipids, and the reduction in glycolysis intermediates.

However, the mRNA level of the PPP enzymes was not altered in the acidosis-adapted PDAC cells (data not shown). This result indicates that there might be post-transcriptional regulation of this pathway.

The increased ATP thus inactivated the AMPK pathway, which is generally considered as a metabolic sensor to suppress cancer. AMPK could initiate a series of downstream pathways, including Hippo. We found that the Hippo pathway effector molecules YAP and TAZ were significantly activated in acidosisadapted PDAC cells. Then, YAP/TAZ bound to transcription factor TEAD4 in nucleus, and facilitated MMP1 expression, resulting in PDAC progression (Fig. 7). Previous studies demonstrated that other MMPs are regulated by acidic condition in different diseases. The expression levels of MMP3 and MMP13 were elevated under the degenerated intervertebral disc-like acidic condition ${ }^{33}$. MMP12 is also increased by extracellular acidosis in systemic sclerosis ${ }^{34}$. Acidic microenvironment induces MMP2, MMP3, MMP9 and MMP13 expression to promote breast or lung cancer progression ${ }^{35}$. MMP1 belongs to 
the zinc-dependent endopeptidase family. It has been proved to be closely relevant to proliferation, migration and invasion in various cancers ${ }^{36}$. MMP1 can also promote the development of pancreatic cancer $^{37}$, even become a potential diagnostic marker for pancreatic cancer ${ }^{38}$. However, there lacks of study on the regulation of MMP1 in the cancer acidic microenvironment. Our study revealed that the expression of MMP1 was increased by acidosis, enriching the research on the regulation of acidic microenvironment on MMP family.

\section{Conclusion}

In summary, the present study showed that metabolic reprogramming promotes metastasis of acidosisadapted PDAC cells via AMPK/YAP/MMP1 axis, enriching the mechanism by which acidic environment promotes tumor progression.

\section{Abbreviations}

PDAC, pancreatic ductal adenocarcinoma; EMT, epithelial mesenchymal transition; MMP1, matrix metalloproteinase 1; MST1/2, Ste20-like kinases 1/2; LATS1/2, large tumor suppressor 1/2; YAP, yes association protein; TEAD, TEA domain; CA9, Carbonic Anhydrase 9; MCT1, monocarboxylate transporter 1; MCT4, monocarboxylate transporter 4; Lamp2, Recombinant Lysosomal Associated Membrane Protein 2; AMP, adenosine monophosphate; F6P, fructose-6-phosphate; F1P, fructose-1-phosphate; FBP, fructose1,6-bisphosphate; G6P, glucose-6-phosphate; PC, phosphatidylcholine; PS, phosphosphingolipids.

\section{Declarations}

\section{Ethics approval and consent to participate}

This study was carried out according to the principles of the Helsinki Declaration and approved by the Ethical Committee of the Second Affiliated Hospital of Chongqing Medical University.

\section{Consent for publication}

All authors have consented to publication of the results presented in this manuscript.

\section{Availability of data and material}

Not applicable.

Competing interests: The authors declare that they have no competing interests.

Funding $\square$ This study was funded by the National Natural Science Fund (No. 81972285), Kuanren Talents Program of the Second Affiliated Hospital of Chongqing Medical University (13-002-011, 13-004-009).

\section{Authors' contributions:}


Conceptualization, Song He and Zhihang Zhou; Data acquisition, Siyuan Chen, Bo Ning, Qingliang Wang, Li Zhou, Zhiji Chen, Linhong Mao, Hongtao Liu; Formal analysis, Siyuan Chen, Bo Ning, Yongyu Chen, Zhihang Zhou; Funding acquisition, Zhihang Zhou; Investigation, Zhihang Zhou, Siyuan Chen, Bo Ning, Jinwen Song, Zhiji Chen,; Methodology, Siyuan Chen, Bo Ning, Li Zhou, Zhiji Chen, Linhong Mao, Hongtao Liu, Zihan Yang; Supervision, Song He and Zhihang Zhou; Validation, Zhihang Zhou, Siyuan Chen, Bo Ning; Visualization, Song He, Zhihang Zhou, Siyuan Chen and Bo Ning. Writing - review \& editing, Siyuan Chen, Zhihang Zhou, Song He, Zihan Yang, and Bo Ning. The author(s) read and approved the final manuscript.

Acknowledgements: We sincerely appreciate all lab members. This study was funded by the National Natural Science Fund (No. 81972285), Kuanren Talents Program of the Second Affiliated Hospital of Chongqing Medical University (13-002-011, 13-004-009).

\section{References}

1. Sun, L., Suo, C., Li, S., Zhang, H. \& Gao, P. Metabolic reprogramming for cancer cells and their microenvironment: Beyond the Warburg Effect. Biochimica et biophysica acta. Reviews on cancer 1870, 51-66, doi:10.1016/j.bbcan.2018.06.005 (2018).

2. Boedtkjer, E. \& Pedersen, S. The Acidic Tumor Microenvironment as a Driver of Cancer. Annual review of physiology 82, 103-126, doi:10.1146/annurev-physiol-021119-034627 (2020).

3. Rohani, N. et al. Acidification of Tumor at Stromal Boundaries Drives Transcriptome Alterations Associated with Aggressive Phenotypes. Cancer research 79, 1952-1966, doi:10.1158/00085472.can-18-1604 (2019).

4. Tian, X. et al. Acidic Microenvironment Up-Regulates Exosomal miR-21 and miR-10b in Early-Stage Hepatocellular Carcinoma to Promote Cancer Cell Proliferation and Metastasis. Theranostics 9 , 1965-1979, doi:10.7150/thno.30958 (2019).

5. Faes, S. et al. Acidic tumor microenvironment abrogates the efficacy of mTORC1 inhibitors. Molecular cancer 15, 78, doi:10.1186/s12943-016-0562-y (2016).

6. Reuss, A., Groos, D., Buchfelder, M. \& Savaskan, N. The Acidic Brain-Glycolytic Switch in the Microenvironment of Malignant Glioma. International journal of molecular sciences 22, doi:10.3390/ijms22115518 (2021).

7. Chen, C. et al. Targeting LIN28B reprograms tumor glucose metabolism and acidic microenvironment to suppress cancer stemness and metastasis. Oncogene 38, 4527-4539, doi:10.1038/s41388-0190735-4 (2019).

8. Zhang, Y. et al. Amino acids and RagD potentiate mTORC1 activation in CD8 T cells to confer antitumor immunity. Journal for immunotherapy of cancer 9, doi:10.1136/jitc-2020-002137 (2021).

9. Wang, D. et al. Cisplatin-resistant NSCLC cells induced by hypoxia transmit resistance to sensitive cells through exosomal PKM2. Theranostics 11, 2860-2875, doi:10.7150/thno.51797 (2021). 
10. Zhou, Z. et al. The acid-sensing ion channel, ASIC2, promotes invasion and metastasis of colorectal cancer under acidosis by activating the calcineurin/NFAT1 axis. Journal of experimental \& clinical cancer research : CR 36, 130, doi:10.1186/s13046-017-0599-9 (2017).

11. Zhou, Z. H. et al. Chromatin accessibility changes are associated with enhanced growth and liver metastasis capacity of acid-adapted colorectal cancer cells. Cell Cycle 18, 511-522, doi:10.1080/15384101.2019.1578145 (2019).

12. Anemone, $\mathrm{A}$. et al. Tumour acidosis evaluated in vivo by MRI-CEST $\mathrm{pH}$ imaging reveals breast cancer metastatic potential. Br J Cancer 124, 207-216, doi:10.1038/s41416-020-01173-0 (2021).

13. Wan, M. et al. Zwitterion-Based Hydrogen Sulfide Nanomotors Induce Multiple Acidosis in Tumor Cells by Destroying Tumor Metabolic Symbiosis. Angew Chem Int Ed Eng/ 60, 16139-16148, doi:10.1002/anie.202104304 (2021).

14. Zhao, M. et al. GSH-dependent antioxidant defense contributes to the acclimation of colon cancer cells to acidic microenvironment. Cell Cycle 15, 1125-1133, doi:10.1080/15384101.2016.1158374 (2016).

15. Corbet, C. et al. The SIRT1/HIF2alpha axis drives reductive glutamine metabolism under chronic acidosis and alters tumor response to therapy. Cancer Res 74, 5507-5519, doi:10.1158/00085472.CAN-14-0705 (2014).

16. Corbet, C. et al. Acidosis Drives the Reprogramming of Fatty Acid Metabolism in Cancer Cells through Changes in Mitochondrial and Histone Acetylation. Cell Metab 24, 311-323, doi:10.1016/j.cmet.2016.07.003 (2016).

17. Schoonjans, C. A. et al. Acidosis-induced metabolic reprogramming in tumor cells enhances the antiproliferative activity of the PDK inhibitor dichloroacetate. Cancer Lett 470, 18-28, doi:10.1016/j.canlet.2019.12.003 (2020).

18. Xu, X. et al. Rewiring of purine metabolism in response to acidosis stress in glioma stem cells. Cell Death Dis 12, 277, doi:10.1038/s41419-021-03543-9 (2021).

19. Shaw, R. J. et al. The LKB1 tumor suppressor negatively regulates mTOR signaling. Cancer Cell 6, 9199, doi:10.1016/j.ccr.2004.06.007 (2004).

20. DeRan, M. et al. Energy stress regulates hippo-YAP signaling involving AMPK-mediated regulation of angiomotin-like 1 protein. Cell Rep 9, 495-503, doi:10.1016/j.celrep.2014.09.036 (2014).

21. Klein, A. P. Pancreatic cancer epidemiology: understanding the role of lifestyle and inherited risk factors. Nat Rev Gastroenterol Hepato/ 18, 493-502, doi:10.1038/s41575-021-00457-x (2021).

22. Kolosenko, I., Avnet, S., Baldini, N., Viklund, J. \& De Milito, A. Therapeutic implications of tumor interstitial acidification. Semin Cancer Bio/ 43, 119-133, doi:10.1016/j.semcancer.2017.01.008 (2017).

23. Ge, T. et al. The Role of the Pentose Phosphate Pathway in Diabetes and Cancer. Front Endocrinol (Lausanne) 11, 365, doi:10.3389/fendo.2020.00365 (2020).

24. Laronha, H. et al. Challenges in Matrix Metalloproteinases Inhibition. Biomolecules 10, doi:10.3390/biom10050717 (2020). 
25. Zhou, Z., Ma, X., Wang, F., Sun, L. \& Zhang, G. A Matrix Metalloproteinase-1 Polymorphism, MMP11607 (1G>2G), Is Associated with Increased Cancer Risk: A Meta-Analysis Including 21,327 Patients. Dis Markers 2018, 7565834, doi:10.1155/2018/7565834 (2018).

26. Ansari, D. et al. The Hippo Signaling Pathway in Pancreatic Cancer. Anticancer Res 39, 3317-3321, doi:10.21873/anticanres.13474 (2019).

27. Lin, K. C., Park, H. W. \& Guan, K. L. Regulation of the Hippo Pathway Transcription Factor TEAD. Trends Biochem Sci 42, 862-872, doi:10.1016/j.tibs.2017.09.003 (2017).

28. Boedtkjer, E. \& Pedersen, S. F. The Acidic Tumor Microenvironment as a Driver of Cancer. Annu Rev Physiol 82, 103-126, doi:10.1146/annurev-physiol-021119-034627 (2020).

29. White, M. A. et al. GLUT12 promotes prostate cancer cell growth and is regulated by androgens and CaMKK2 signaling. Endocr Relat Cancer 25, 453-469, doi:10.1530/ERC-17-0051 (2018).

30. Shi, Y. et al. Let-7a-5p inhibits triple-negative breast tumor growth and metastasis through GLUT12mediated warburg effect. Cancer Lett 495, 53-65, doi:10.1016/j.canlet.2020.09.012 (2020).

31. Jiang, P., Du, W. \& Wu, M. Regulation of the pentose phosphate pathway in cancer. Protein Cell 5, 592602, doi:10.1007/s13238-014-0082-8 (2014).

32. Li, Q. et al. Rac1 activates non-oxidative pentose phosphate pathway to induce chemoresistance of breast cancer. Nat Commun 11, 1456, doi:10.1038/s41467-020-15308-7 (2020).

33. Li, H., Liu, H., Zhang, N. \& Zhu, Z. Involvement of the G-Protein-Coupled Receptor 4 in the Increased Expression of RANK/RANKL/OPG System and Neurotrophins by Nucleus Pulposus Cells under the Degenerated Intervertebral Disc-Like Acidic Microenvironment. Biomed Res Int 2020, 1328436, doi:10.1155/2020/1328436 (2020).

34. Andreucci, E. et al. Glycolysis-derived acidic microenvironment as a driver of endothelial dysfunction in systemic sclerosis. Rheumatology (Oxford), doi:10.1093/rheumatology/keab022 (2021).

35. Nakanishi, M. et al. Acidic microenvironment induction of interleukin-8 expression and matrix metalloproteinase-2/-9 activation via acid-sensing ion channel 1 promotes breast cancer cell progression. Oncol Rep 45, 1284-1294, doi:10.3892/or.2020.7907 (2021).

36. Liu, H. et al. Circular RNA circDLC1 inhibits MMP1-mediated liver cancer progression via interaction with HuR. Theranostics 11, 1396-1411, doi:10.7150/thno.53227 (2021).

37. Chen, Y. et al. MicroRNA hsa-miR-623 directly suppresses MMP1 and attenuates IL-8-induced metastasis in pancreatic cancer. Int J Onco/ 55, 142-156, doi:10.3892/ijo.2019.4803 (2019).

38. Xie, J. et al. Identification of potential diagnostic biomarkers in MMPs for pancreatic carcinoma. Medicine (Baltimore) 100, e26135, doi:10.1097/MD.0000000000026135 (2021).

\section{Tables}

Table 1. The correlation between MMP1 expression level and clinicopathological parameters 
Features

No. of patients $\quad$ MMP1 expression status

(\%)

Low $(n=41)$ No.patient High $(n=21)$ No.patient

(\%)

(\%)

Gender

0.410

Male

24(38.7)

14(58.3)

10(41.7)

Female

38(61.3)

27(71.1)

11(28.9)

Age

$\leq 59$

$34(54.8)$

21(61.8)

13(38.2)

$>59$

28(45.2)

20(71.4)

8(28.6)

T stage

$1+2$

47(75.8)

35(74.5)

12(25.5)

0.026

$3+4$

15(24.2)

6(40.0)

$9(60.0)$

$\mathrm{N}$ stage

0

42(67.7)

27(64.3)

15(35.7)

1

16(25.8)

13(81.2)

3(18.8)

2

4(6.5)

1(25.0)

$3(75.0)$

M stage

$\begin{array}{llll}0 & 61(98.4) & 40(65.6) & 21(34.4) \\ 1 & 1(1.6) & 1(100) & 0(0)\end{array}$

0.095

TNM stage

\begin{tabular}{llll} 
I & $31(50.0)$ & $24(77.4)$ & $7(22.6)$ \\
\hline II & $26(41.9)$ & $14(53.8)$ & $12(46.2)$ \\
\hline III & $4(6.5)$ & $2(50.0)$ & $2(50.0)$ \\
IV & $1(1.6)$ & $1(100)$ & $0(0)$
\end{tabular}

Vessel invasion

without

34(54.8)

24(70.6)

10(29.4)

with

28(45.2)

17(60.7)

11(39.3)

Nerve invasion

without

29(46.8)

23(79.3)

6(20.7)

with

33(53.2)

18(54.5)

15(45.5) 
Differentiation degree

\begin{tabular}{llll} 
Poor & $20(32.3)$ & $13(65.0)$ & $7(35.0)$ \\
\hline Moderate & $27(43.5)$ & $20(74.0)$ & $7(26.0)$ \\
\hline Well & $15(24.2)$ & $8(53.3)$ & $7(46.7)$
\end{tabular}

CA9 expression

0.018

$\begin{array}{llll}\text { low } & 43(69.4) & 33(76.7) & 10(23.3) \\ \text { high } & 19(30.6) & 8(42.1) & 11(57.9)\end{array}$

ASIC1 expression

0.013

\begin{tabular}{llll} 
low & $25(40.3)$ & $21(84.0)$ & $4(16.0)$ \\
\hline high & $37(59.7)$ & $20(54.1)$ & $17(45.9)$
\end{tabular}

ASIC2 expression

\begin{tabular}{llll} 
low & $41(66.1)$ & $32(78.0)$ & $9(22.0)$ \\
\hline high & $21(33.9)$ & $9(42.9)$ & $12(57.1)$
\end{tabular}

61 PDAC clinical samples were used to evaluate the correlation between MMP1 expression and clinicopathological features. The bold values indicated that the P value was smaller than 0.05 .

Figures 



e

PANC1-increased

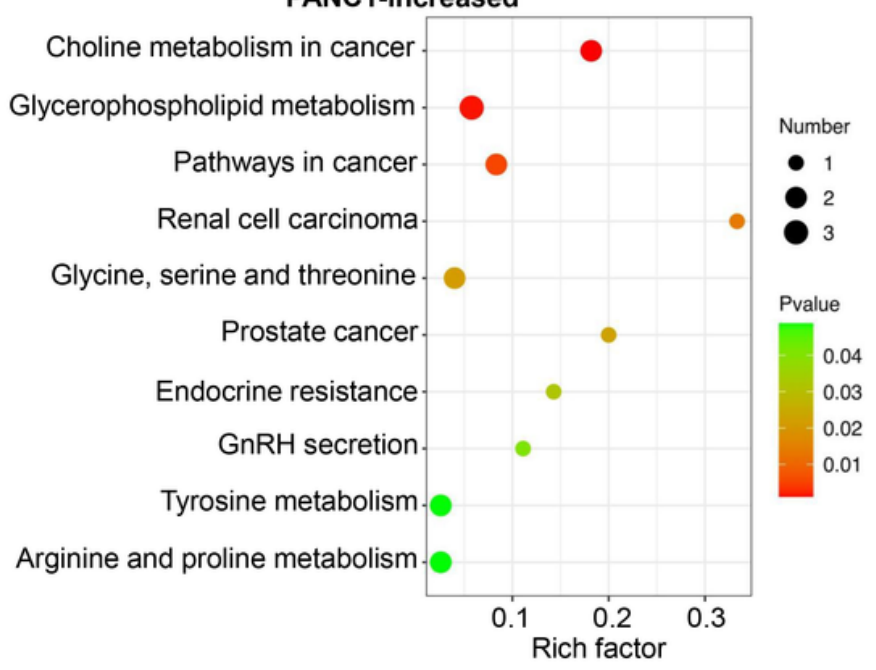

g

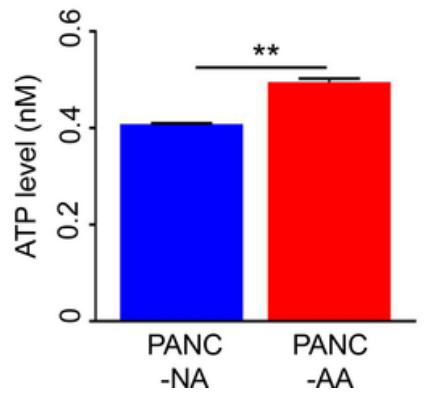

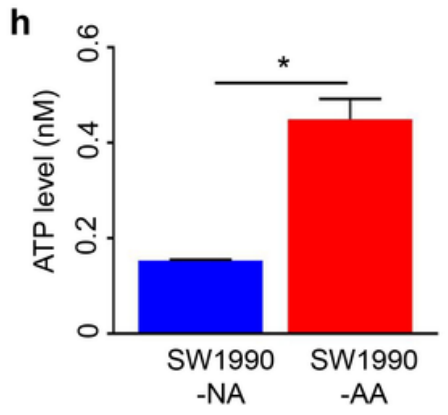

f

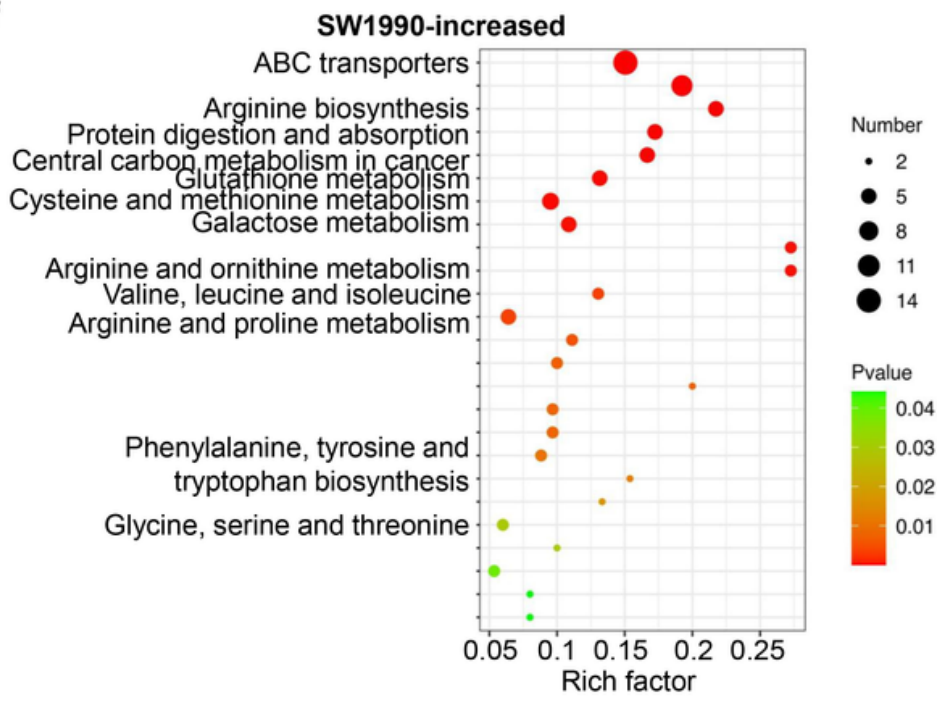

i

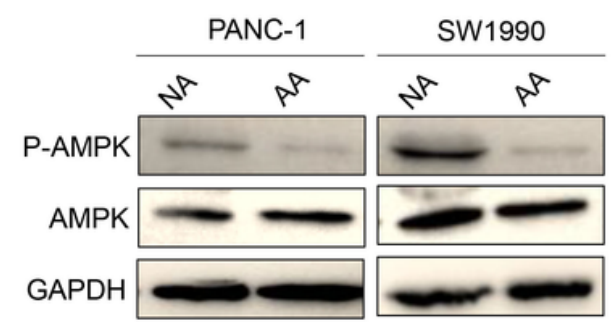

\section{Figure 1}

Acidosis-adapted cells inhibited AMPK activation through metabolic changes. a. The GC-MS detected changes of metabolites in SW1990-NA and SW1990-AA cells. b. The LC-MS revealed changes of metabolites in SW1990-NA and SW1990-AA cells. c\&d. Analysis of decreased and increased metabolites in PANC-1-AA vs. SW1990-AA cells. e\&f. Pathway changed in PANC-1-AA and SW1990-AA cells. g\&h. ATP increased in PANC-1-AA and SW1990-AA cells. i. The activation of AMPK was detected by WB in acidosisadapted PDAC cells and normal cultured PDAC cells. AMP, adenosine monophosphate; F6P, fructose-6phosphate; F1P, fructose-1-phosphate; FBP, fructose-1,6-bisphosphate; G6P, glucose-6-phosphate; PC, phosphatidylcholine; PS, phosphosphingolipids. *, $\mathrm{P}<0.05 ; * \star, \mathrm{P}<0.01 ; * \star \star, P<0.001 ; * \star \star \star, P<0.0001 \rrbracket \mathrm{N}=3$. 




C
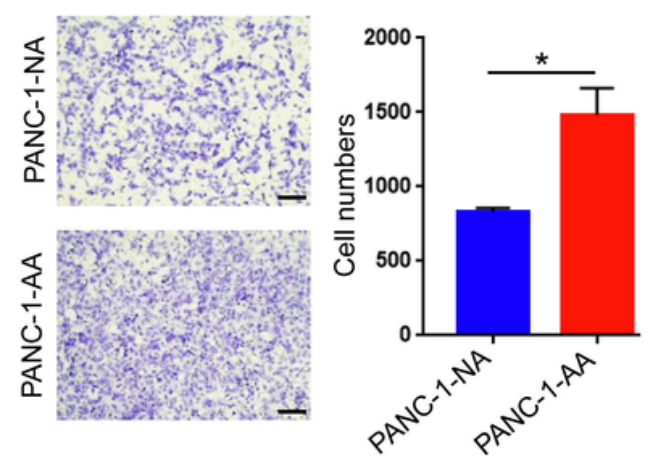

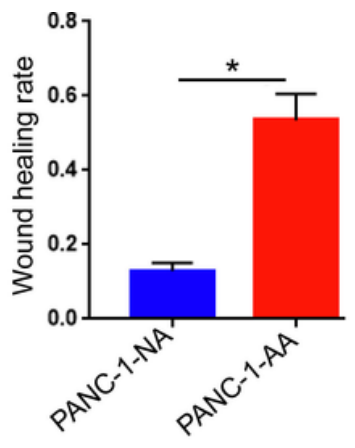

d b

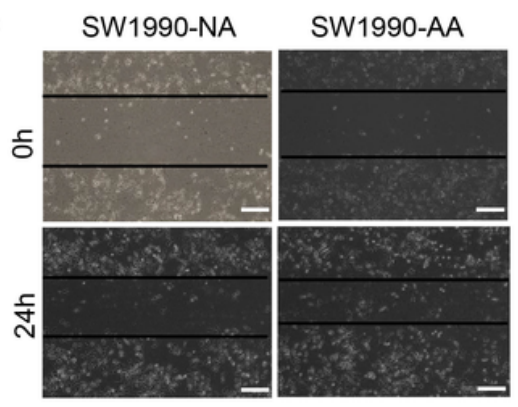

e
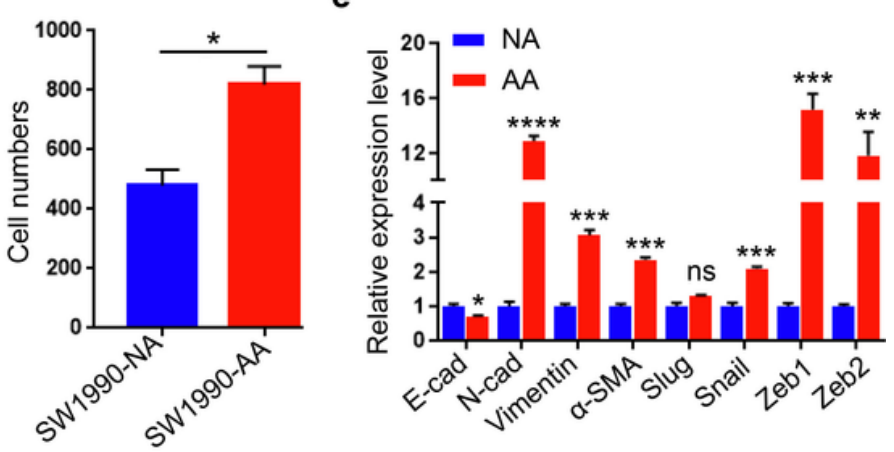

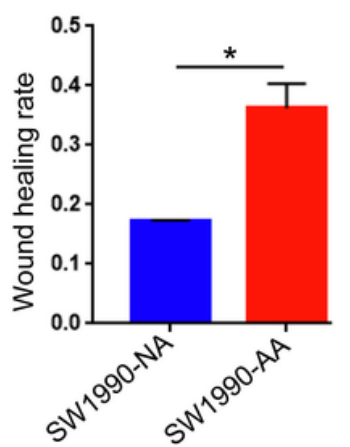

5

\section{(n)}

g

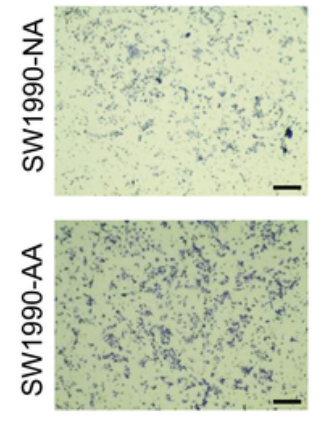

PANC-1-NA
PANC-1-AA

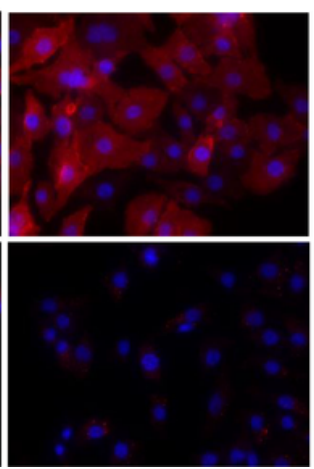

SW1990-NA

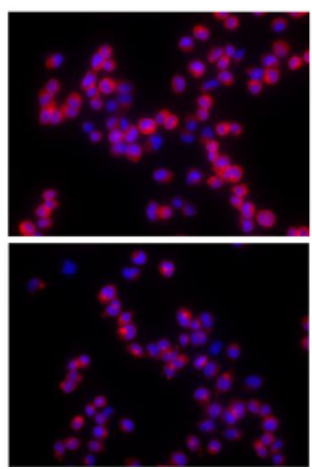

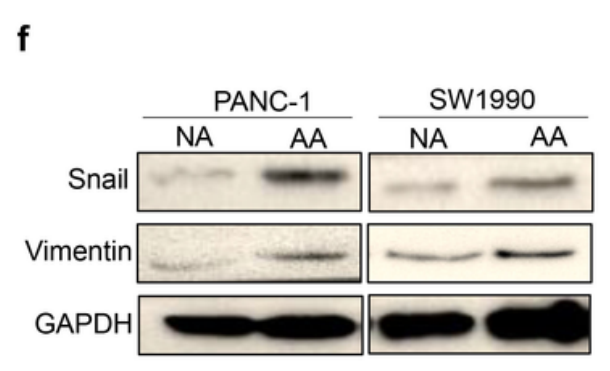

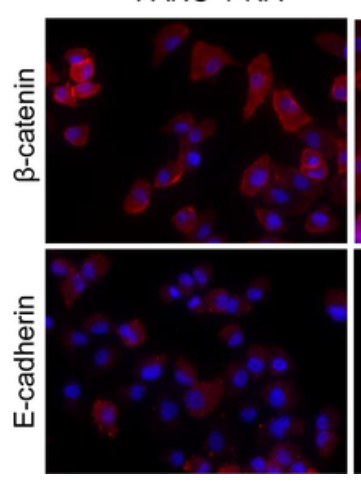

SW1990-AA

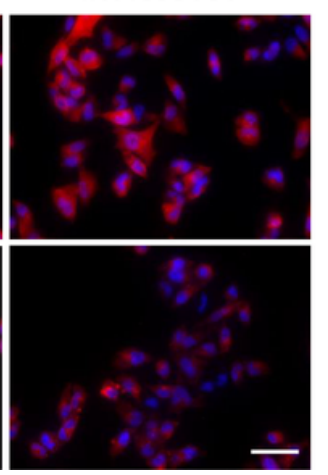

Figure 2

Migration and invasion capacity were enhanced in acid-adapted PDAC cell. a\&b. Wound healing assays reflected migration in PANC-1-AA, SW1990-AA and NA cells. c\&d. Transwell assay showed invasion abilities in PANC-1-AA and SW1990-AA and NA cells. The scale bars on the lower right are 200 $\mu \mathrm{m}$. e. mRNA levels of EMT markers (E-cadherin, N-cadherin, Vimentin, a-SMA, Slug, Snail, Zeb1 and Zeb2) were detected in acid-adapted PDAC and normal cells by qPCR. $f$. WB detected protein levels of Snail and Vimentin in PANC-1-AA and SW1990-AA cells. g. Immunofluorescence detection of E-cadherin and $\beta$ catenin in PANC-1-AA and SW1990-AA cells. Nuclei were stained in blue with DAPI. The white scale bars

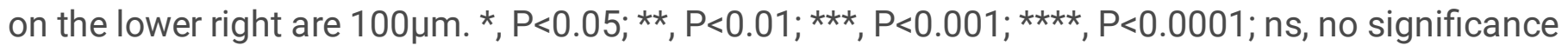
difference; $\mathrm{N}=3$. 
a

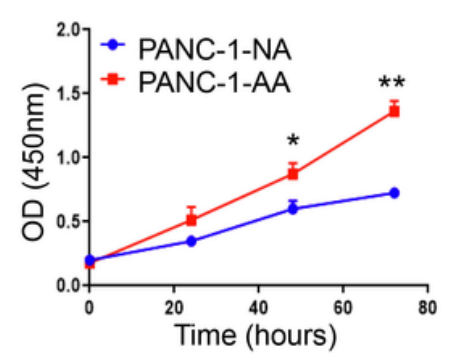

e

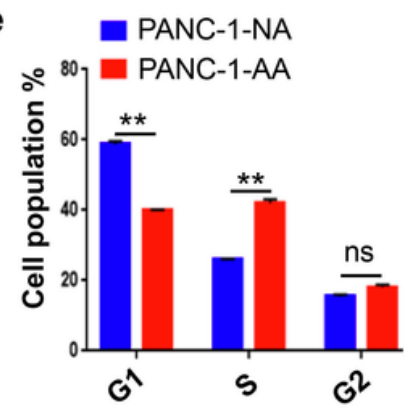

f

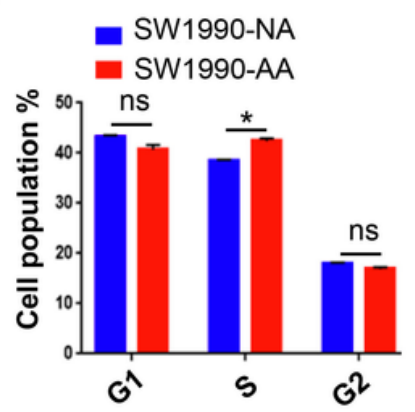

b

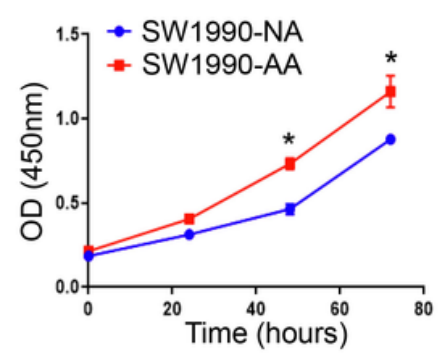

g
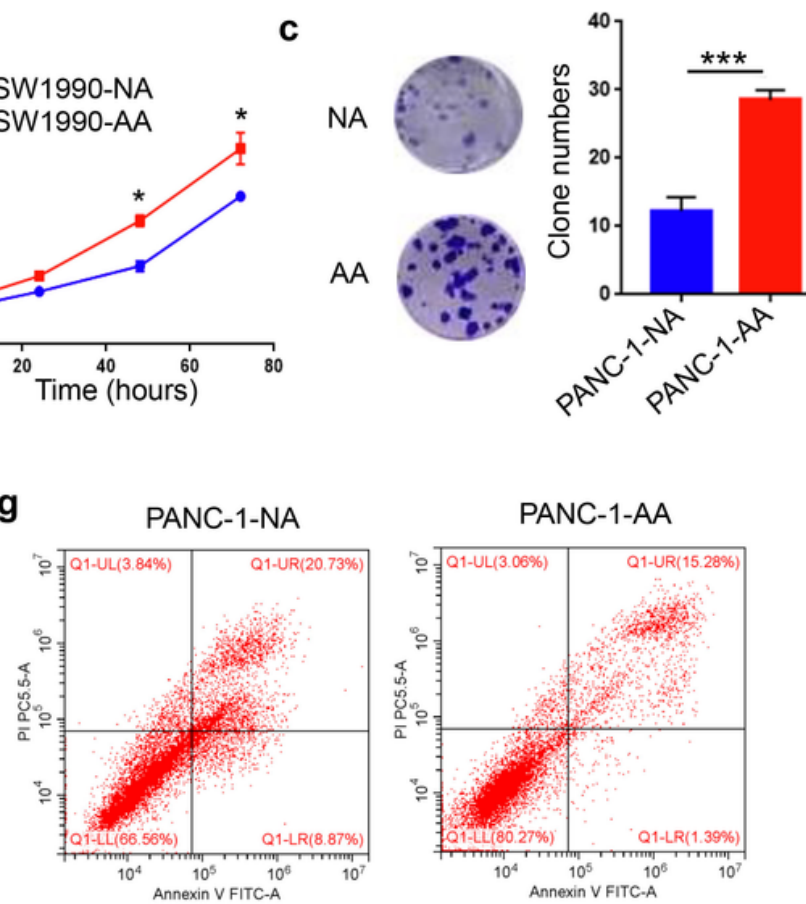

d
AA

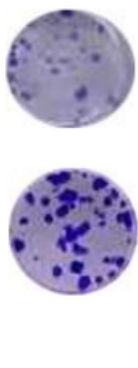

NA
NA
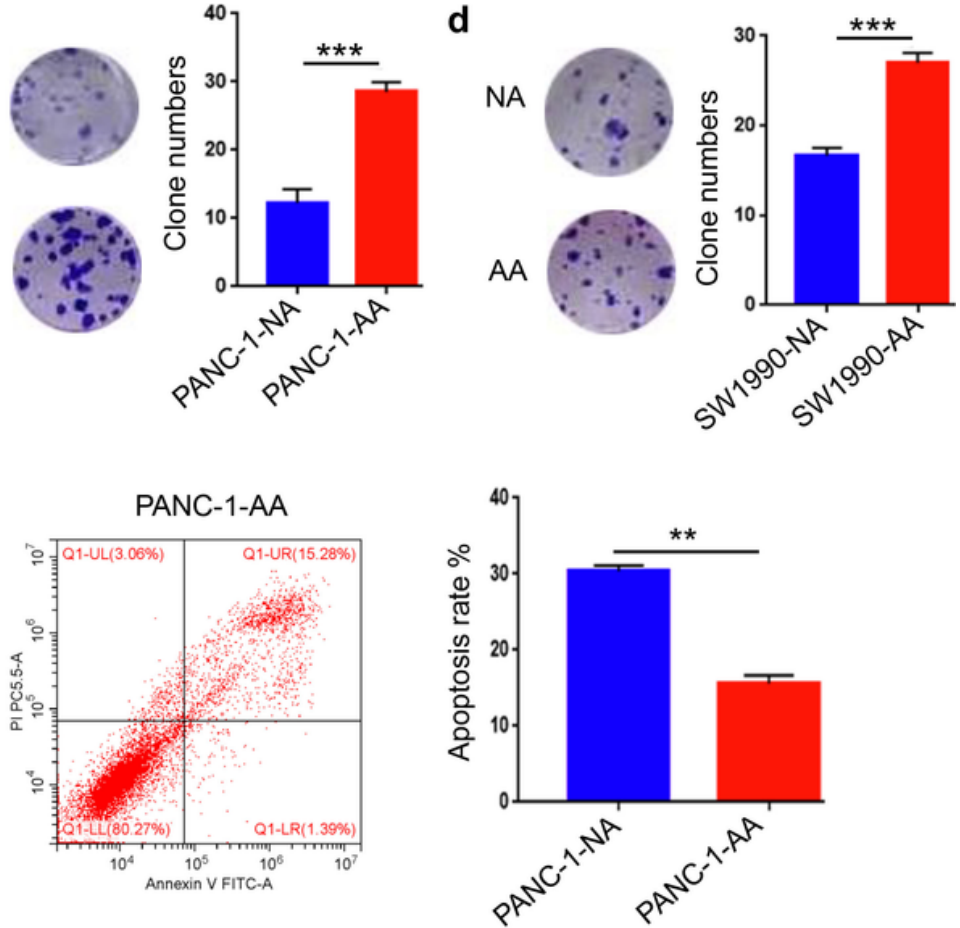

h
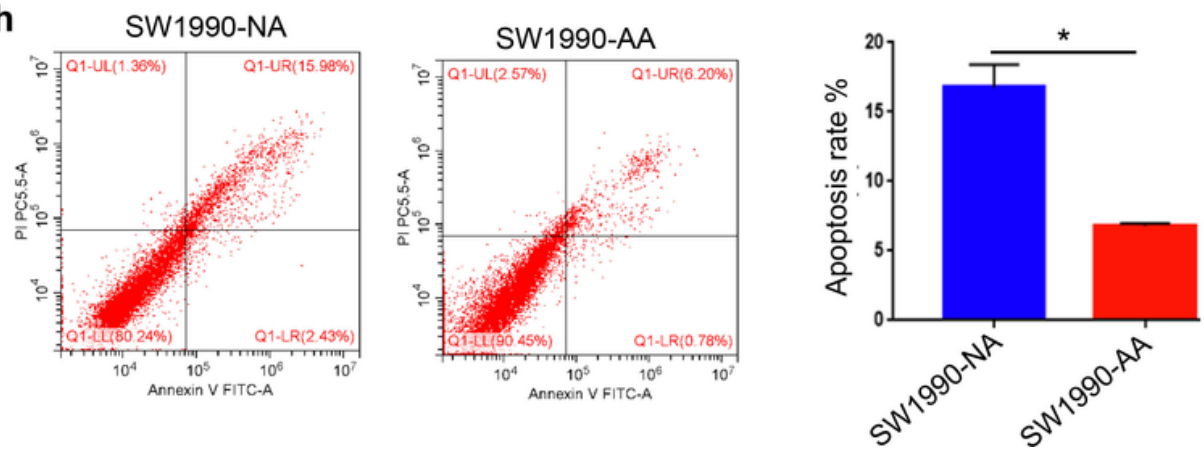

Figure 3

Proliferation was increased in acid-adapted PDAC cell. a\&b. CCK8 assays showed the PDAC cells proliferation in PANC-1-AA and SW1990-AA cells under acidic medium and their parental cells under normal medium. c\&d. Effects of acidic environment on clonogenicity of PANC-1 and SW1990 cells. e\&f. Summary data of cell cycle analysis by flow cytometry in acid-adapted PDAC cells and normal cells. g\&h. Cell apoptosis analysis by flow cytometry in PANC-1-AA and SW1990-AA cells (left) and summary data (right). *, $\mathrm{P}<0.05 ; * \star, \mathrm{P}<0.01 ; * \star \star, P<0.001 ; * \star \star \star, P<0.0001 ; n s$, no significance difference; $\mathrm{N}=3$. 
a

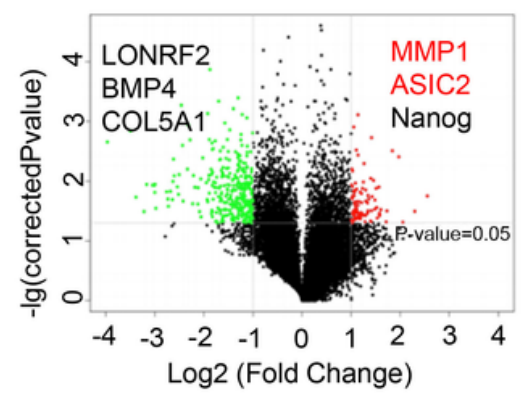

b

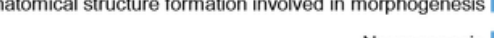

Neurogenesis

Cell adhesion

Extracellular structure organization

Biological adhesion

Extracellular matrix organization

Extracellular matrix component

- Biological Process
"- Cellular Component $\quad$ Generation of neurons

- Molecular Function Nervous system development

$0-4 m \ln ^{-4}$

- $\log ($ Corrected p-Value) c

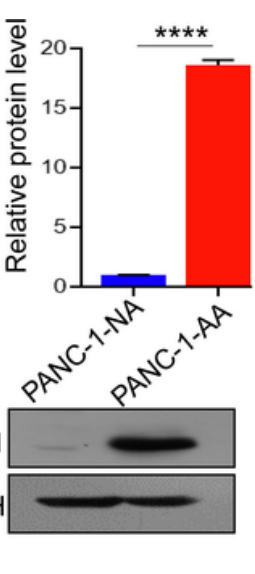

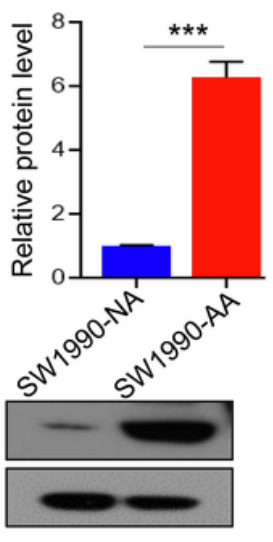

d

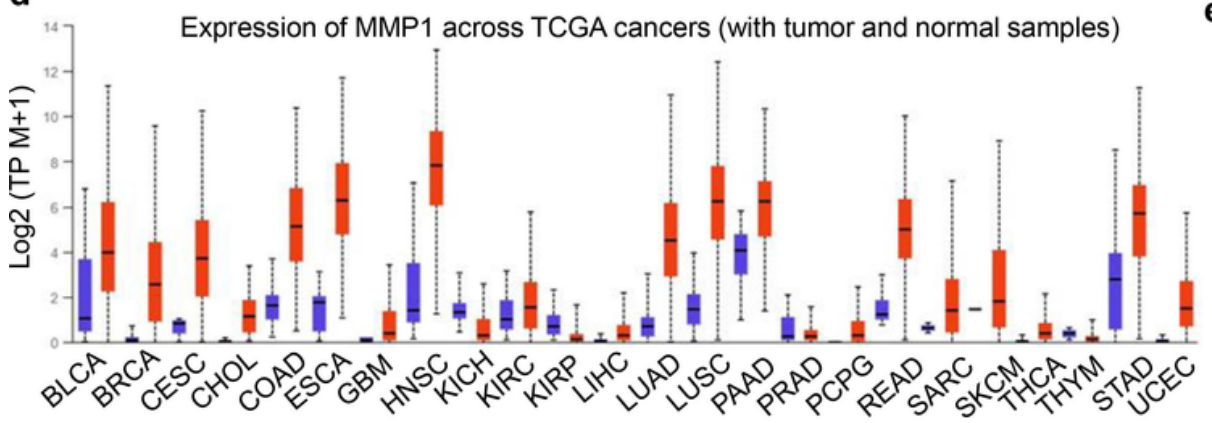

e

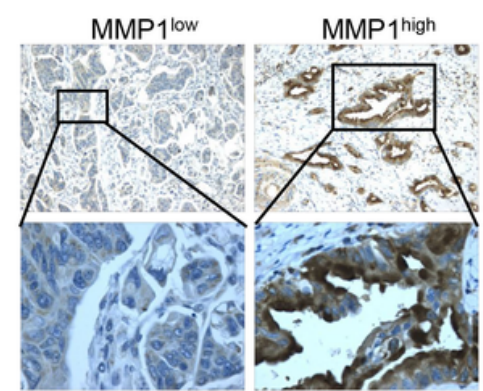

Normal

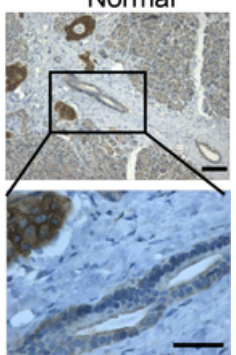

$f$

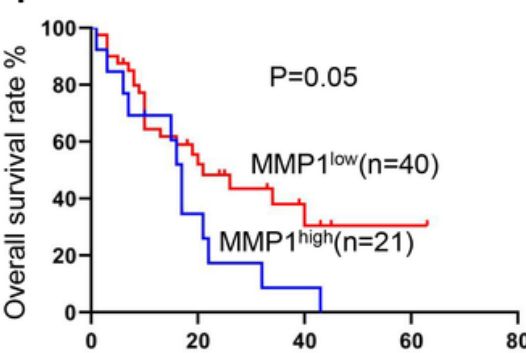

Time after surgery (months) g

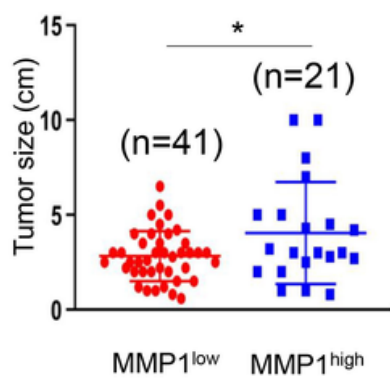

h



i

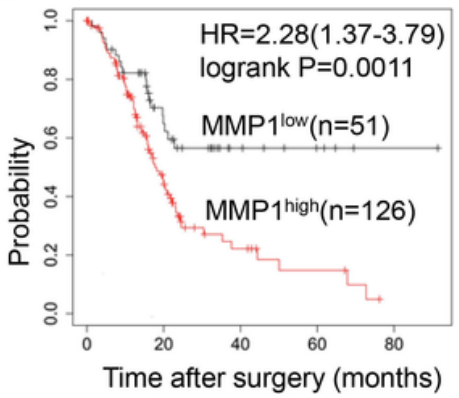

\section{Figure 4}

MMP1 expression level was increased in acid-adapted PDAC cell. a\&b. Results of High throughput microarray profiling. c. Transcription level and protein level of MMP1 were detected by qPCR and WB in acid-adapted PDAC cells and normal cells. d. Expression of MMP1 mRNA in various tumors from TCGA database. e. Representative images of immunohistological staining on MMP1 proteins in PDAC tissues ( $n=61$ patients). The scale bars on the lower right are 200 $\mu \mathrm{m}$ (the upper panel) and 100 $\mu \mathrm{m}$ (the lower panel). f. Analysis of survival rates in PDAC patients with low and high MMP1 expression level ( $n=61$ patients). g. Relationship between the MMP1 protein expression level and the size of pancreatic cancer. $h$. Expression of MMP1 in different tumor stages from TCGA database. i. Analysis of prognosis of patients with high expression of MMP1 and low expression of MMP1 by Kaplan-Meier from KM plotter. * ${ }^{*}<0.05$; $\star \star, P<0.01 ; * \star \star, P<0.001 ; * \star \star *, P<0.0001 ; n s$, no significance difference $\varangle \mathrm{N}=3$. 
a

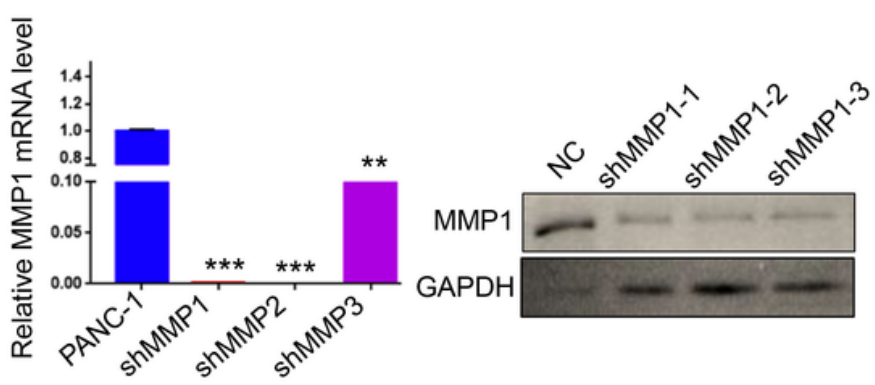

b

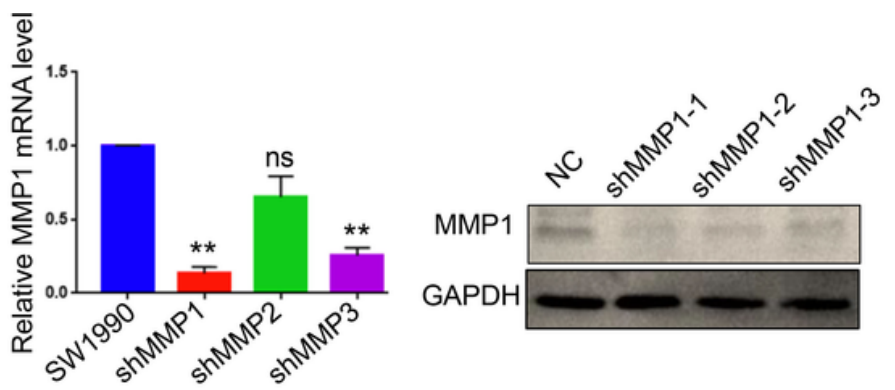

C
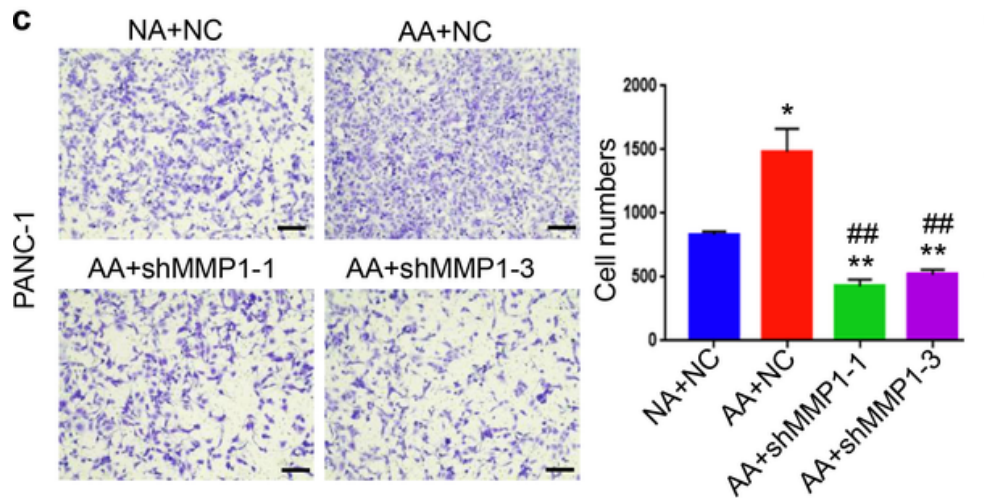
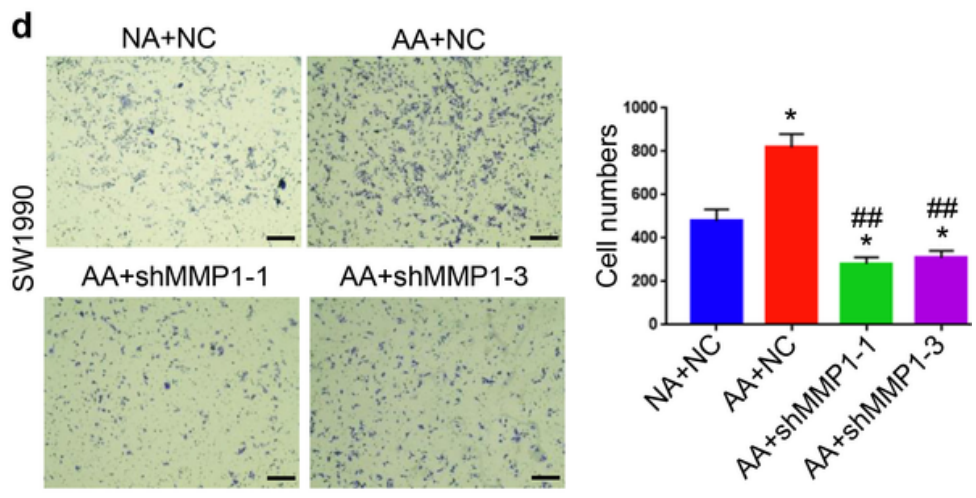

e
$\stackrel{ }{\circ}$

$N A+N C$

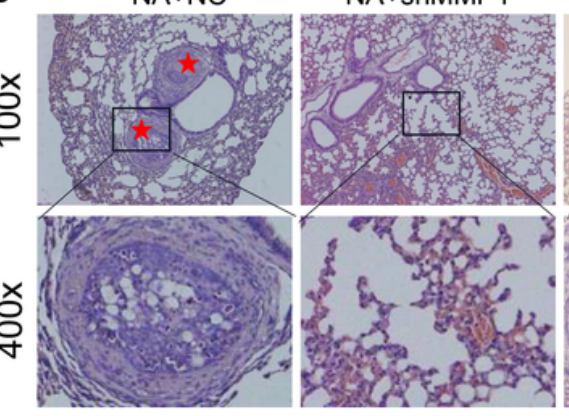

$\mathrm{AA}+\mathrm{NC}$

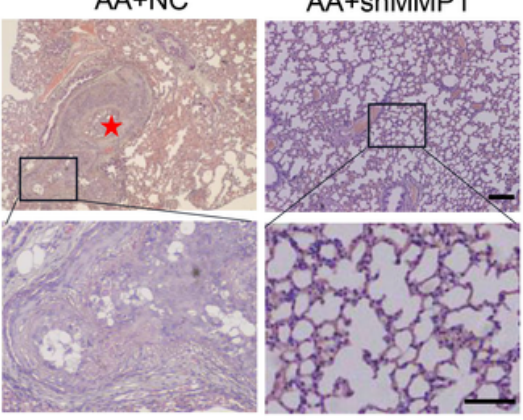

$\mathbf{f}$

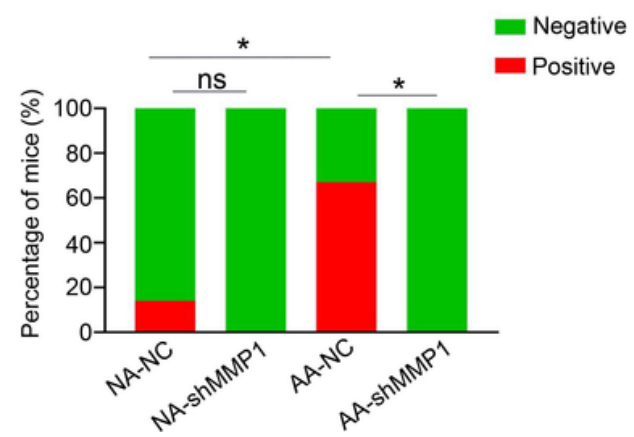

Figure 5

Knockdown of MMP1 impaired acid-adapted PDAC cell invasion. a\&b. mRNA levels (left) and protein levels (right) of MMP1 detected by qPCR and WB in MMP1-knockeddown PANC-1 and SW1990 cells. c\&d. PDAC cell invasion presented as original images and presented as summary data in MMP1-knockdown PANC-1-AA and SW1990-AA cells. The scale bars on the lower right are $200 \mu \mathrm{m}$. e. Representative images of $H \& E$ staining of tail vein injection models. $f$. Summary data of tumor numbers in tail vein injection

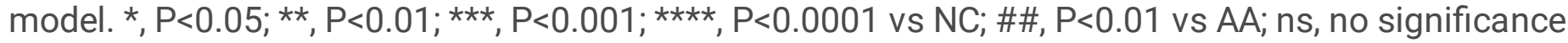
difference $\mathbb{N}=3$. 
Significant Enriched Pathway Terms (Top 20)

Extracellular matrix organization Non-integrin ECM interactions

Gene expression by SREBF

Agrin in postsynaptic differentiation

Beta1 integrin interactions

Right ventricular cardiomyopathy

Cholesterol biosynthesis Axon guidance ECM-receptor interaction Laminin interactions Prion pathway

Cholesterol biosynthesis by SREBP Elastic fibre formation Rho GTPase cycle Signaling by Rho GTPases Basal cell carcinoma L1CAM interactions Beta3 integrin interactions Elastic fibres

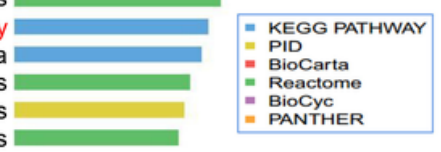

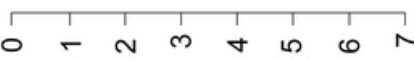

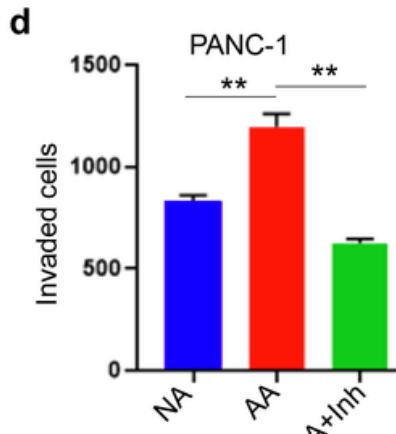
- $\log ($ Corrected p-Value)

e

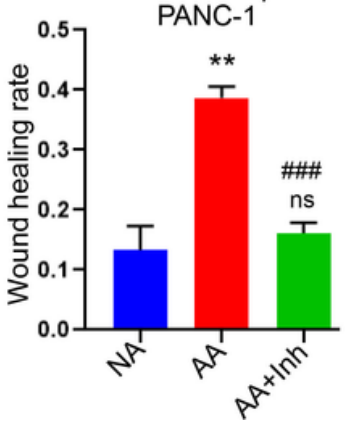

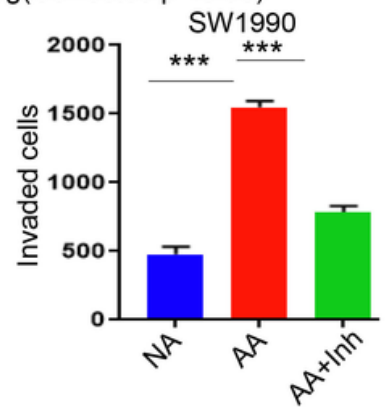

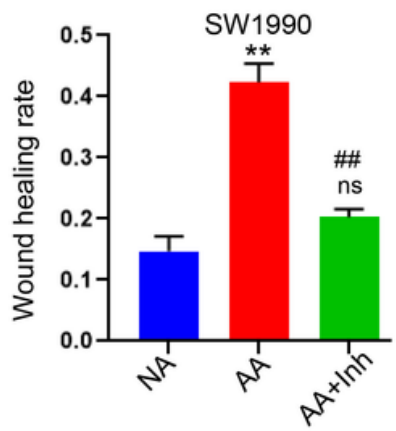

b

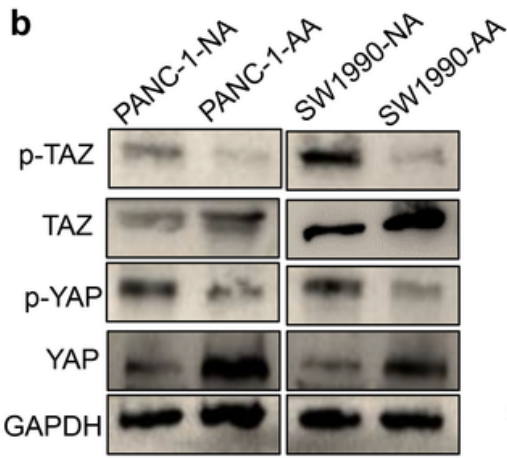

f

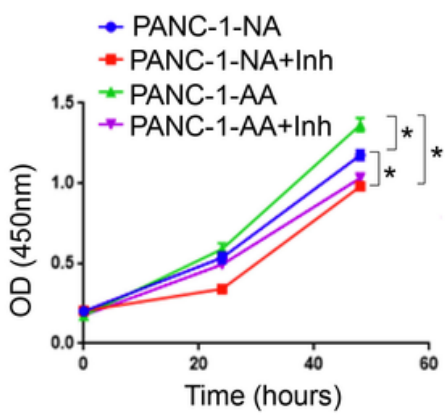

g
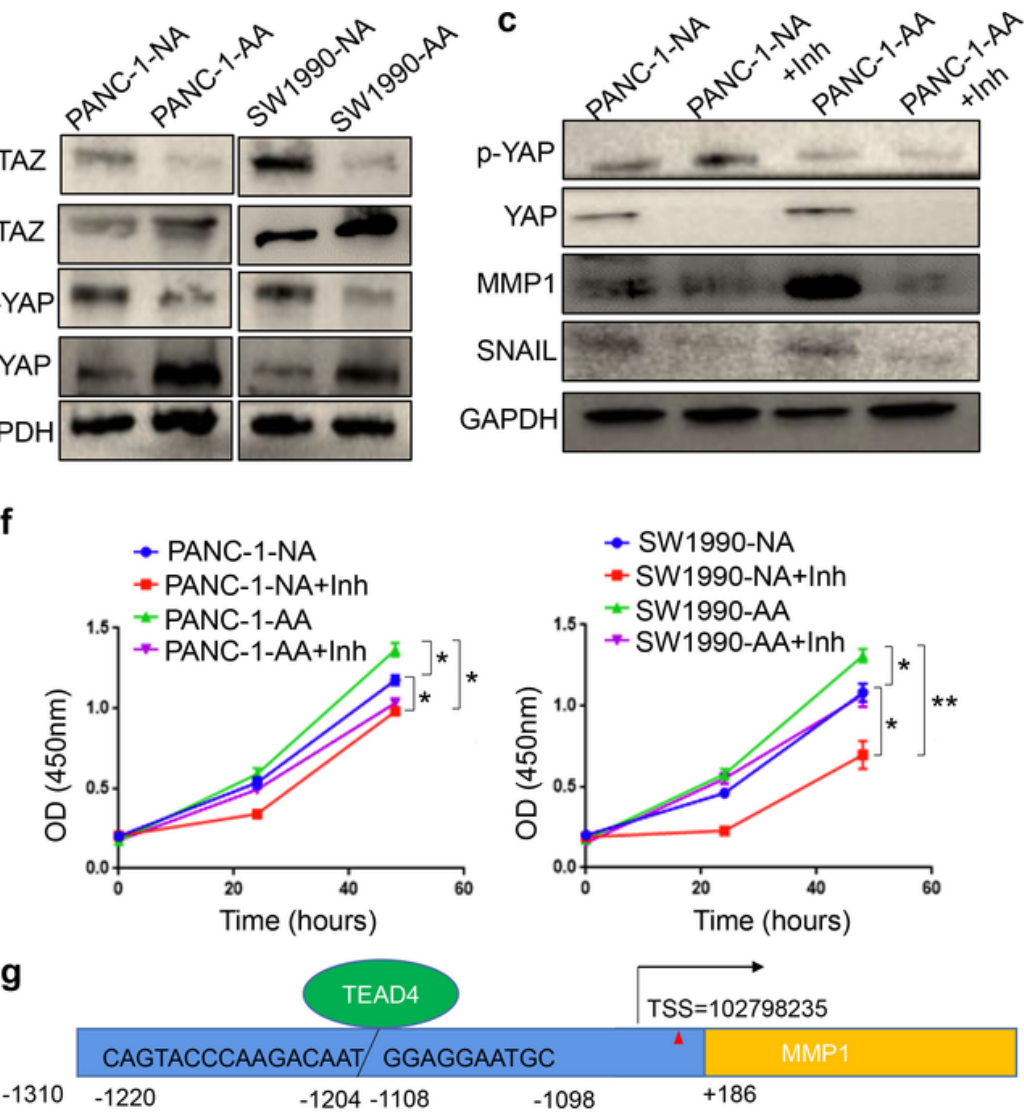

- SW1990-NA+Inh

- SW1990-AA

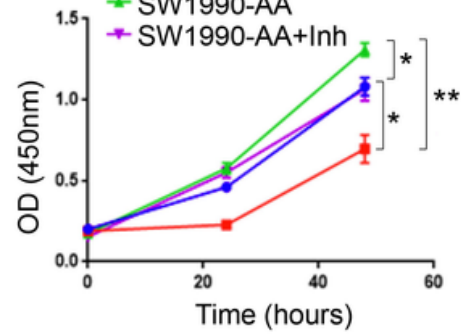
Promoter=102796925-102798421 Genome=hg38;chr11-:102798049-102799545

h

PANC-1 SW1990

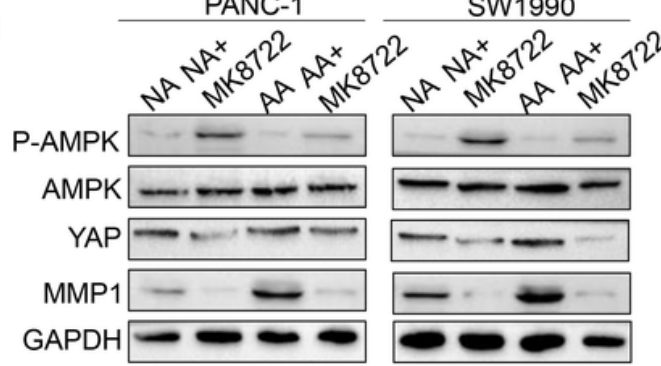

Figure 6

Inactivation of Hippo pathway mediated MMP1 induction in acid-adapted cells. a. KEGG analysis of the differentially expressed genes showed that Hippo pathway was significantly changed in acid-adapted PDAC cells. b. Protein levels were detected by WB of YAP and TAZ in PANC-1-AA and SW1990-AA cells. c.

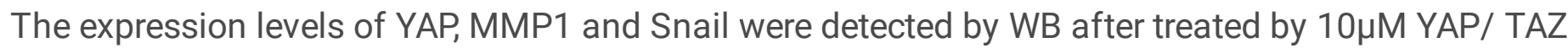
inhibitor ML-7 for 24h. d. Summary data of transwell after treated by YAP/TAZ inhibitor in PANC-1 and SW1990. e. Summary data of wound healing assays after treated by YAP/ TAZ inhibitor in PANC-1 and SW1990 cell. f. Cell proliferation was detected by CCK8 after treated by YAP/ TAZ inhibitor. g. MMP1 transcription factor prediction. $\mathrm{h}$. WB detected P-AMPK, AMPK, YAP, MMP1 protein expression after treated with AMPK inhibitor MK8722 at $1 \mu \mathrm{M}$ for 24h. *, $\mathrm{P}<0.05$; **, $\mathrm{P}<0.01$; ***, $\mathrm{P}<0.001$ vs NC; \#\#, $\mathrm{P}<0.01$, $\# \# \#, P<0.001$ vs $A A ; n s$, no significance difference $\mathbb{N}=3$. 




Figure 7

Proposed mechanisms of acidosis promoted PDAC progression. In acidosis-adapted PDAC cells, glucose enters the cells through GLUT12 transporter, which increases glucose-6-phosphate (G6P) and pentose phosphate pathway (PPP) metabolism, thus affecting amino acids or phosphatidylcholine (PC), phosphosphingolipids (PS) and others metabolism, increased ATP content and inhibited AMPK, so as to activate YAP/ TAZ through AMOTL1. YAP/TAZ translocated into nucleus and bound with TEAD4 to promote MMP1 transcription and increase expression level of MMP1, leading to PDAC cells metastasis and proliferation.

\section{Supplementary Files}

This is a list of supplementary files associated with this preprint. Click to download.

- FigS1.jpg 
- FigS2.jpg

- FigS3.jpg

- FigS4.jpg

- figS5.jpg

- figS6.jpg

- Supplementarytable.docx 